\title{
Commentary on "How Stable is Pitch Labeling Accuracy in Absolute Pitch Possessors?" by W. Gruhn, R. Ristmägi, P. Schneider, A. d'Souza, \& K. Kiilu
}

\author{
KATHRIN BETTINA SCHLEMMER \\ Catholic University Eichstätt-Ingolstadt
}

\begin{abstract}
This paper is a brief commentary on the paper "How stable is pitch labeling accuracy in absolute pitch possessors" by W. Gruhn, R. Ristmägi, P. Schneider, A. d'Souza, and K. Kiilu. The original paper presents evidence for the influence of timbre on the pitch labeling accuracy of absolute pitch (AP) possessors. The presented data result from a solid methodology and show a clear pitch labeling accuracy drop from instrumental timbres to sinusoidal tones, with manipulated sounds being in the middle. The commentary underlines the view that the presented results are compatible with a memory perspective on AP and can be interpreted as evidence for the influence of training on the development of AP.
\end{abstract}

Submitted 2018 November 21; accepted 2019 February 07.

KEYWORDS: absolute pitch, pitch labeling accuracy

ABSOLUTE pitch (AP), defined as the ability to label individual pitches upon hearing, or to produce pitches without external references, has interested music psychological research for more than a century, and both the development of the skill as well as its behavioral and neural characteristics are issues still in the process of academic discussion.

Concerning the characterization of AP, two questions have been addressed, namely whether AP possessors and non-possessors differ in their perceptual processing and in their cognitive representation of pitches. As to the former, there is currently no evidence for a finer discrimination of pitches among AP possessors compared to non-possessors (see Deutsch, 2013, for an overview), but there is some evidence that AP is partly explained by the hypothesis of categorical perception (Elmer, Rogenmoser, Kühnis, \& Jäncke, 2015). Concerning the cognitive processing of pitches, research by Rakowsky and MorawskaBüngeler (1987) has shown that AP can be described as an excellent long-term memory for individual pitches, while Rogenmoser, Elmer, and Jäncke (2015) suggested that by labeling pitches, AP possessors can reduce the processing load in working memory. Levitin (1994) proposed that AP processing consists of two distinct memory processes, namely pitch memory (which is more common), and pitch labeling (which is exclusive for AP possessors). Recently, Elmer et al. (2015) suggested that the obvious gap between the perceptual and cognitive perspectives on AP might be overcome by neuropsychological research focusing on the functional connectivity of the brain. Specifically, they propose a tight coupling mechanism between tonal inputs and corresponding memory representations among AP possessors during pitch processing, while conceding that influences of genetic and learning factors on this mechanism have yet to be determined.

The paper presented by Gruhn, Ristmägi, Schneider, d'Souza, and Kiilu focuses on perceptual aspects of AP and summarizes much of the neuroscientific evidence concerning AP perception. Specifically, the authors analyze the influence of an aural orientation as holistic or spectral listeners (Schneider \& Wengenroth, 2009) on pitch labeling. Possible influences of individual perceptual or cognitive styles on AP are an interesting line of research which has previously been addressed, for example by Chin (2003), who suggested that an analytical cognitive style together with a tendency towards a narrow focus of attention support the acquisition of AP among early music learners. Chin referred to the high occurrence rate of AP among autistic people whose cognitive style can be characterized by an extreme concentration on details. Following up on this research and investigating professional musicians, Schlemmer (2009) found no evidence that AP possessors could be characterized by a field independent 
cognitive style (a concept closely related to the analytical cognitive style) while handling visual and musical tasks. Since the potential influence of listening orientation or perceptual style is not yet resolved, the paper by Gruhn et al. adds important information on this topic.

The central issue of the paper by Gruhn et al. is the influence of psychoacoustic parameters on the accuracy of pitch labeling. There is a body of existing research on the difference in pitch labeling accuracy when instrumental timbres and sinusoidal tones are used (Marvin \& Brinkman, 2000; Miyazaki, 1989, 1990; Schlemmer, Kulke, Kuchinke, \& Van der Meer, 2005), so in my opinion, the most interesting contribution of the paper concerning this issue is the additional focus on manipulated sounds.

The paper by Gruhn et al. presents the following results regarding the influence of psychoacoustic parameters on the accuracy of pitch labeling: While there is a clear difference in the pitch labeling ability of full AP possessors and partial AP possessors, with the former showing a better performance in all experimental conditions, both groups' performance depended on the timbres of the tones presented. Both full and partial AP possessors were better at labeling tones presented with instrumental timbres than with manipulated timbres, and the lowest accuracy was achieved in the condition with sinusoidal tones. This result confirms previous research regarding sinusoidal tones (Miyazaki, 1989), and extends existing knowledge to manipulated sounds. A related result concerns key colors, with an effect of key color which resembles that of previous studies (Marvin \& Brinkman, 2000; Miyazaki, 1990, Schlemmer et al., 2005). A strength of the paper by Gruhn et al. is certainly the discussion section on the interpretation of the low recognition rates for pure tones among AP possessors.

Concerning differences across participants, the paper by Gruhn et al. shows that pianists are less influenced by different timbres or manipulations, and only show decreased pitch labeling accuracy when being presented with sinusoidal tones. On the other hand, melodic instrument players showed an overall higher hit rate in all conditions, although the influence of timbre was larger on them compared to the pianists. Those participants with an earlier beginning of music instruction showed better performance, which is in line with existing research (e.g. Sergeant, 1969). There was no dominance of one perception mode for AP possessors in a larger sample referred to by the authors, although Figure 7 suggests that the AP possessors tested here tend towards a holistic perception mode, possibly because many of them were pianists. The issue of a possible connection between AP and perceptual styles could not be resolved with the presented data and will need larger and more diverse samples regarding instrumental background for clearer results.

While Gruhn et al. offer convincing arguments concerning the specific influence of psychoacoustic parameters, I do not wholly agree with their interpretation concerning the effects of genetics and training for the development of AP. The authors suggest that both effects may interact, but can also occur independently of each other. Only recently, Loui (2016) has convincingly suggested the use of a diathesis stress model adapted from clinical research for tying together the existing evidence about the development of AP: "It is likely that AP is caused by the convergence of the two pathways of influence: disposition (or vulnerability) and exposure (or experience)" (Loui, 2016, p. 90). Genetic factors as well as family history would be among the dispositional factors, while language background or musical training fall among the developmental factors (which are most powerful within a critical period). Loui suggests that "a combination of at least one predisposition and at least one developmental factor is required for the neural determinants of AP" (Loui, 2016, p. 91). If such a model is used, we should question the authors' notion about the potential independence of genetic and learned factors, because in any way, there seems to be no possibility of developing the skill without learning. Clearly, results concerning the effects of timbre and key color on pitch labeling accuracy can be interpreted as evidence for training effects. Since the most likely way for AP development to take place is the interaction of genetic and learned factors, the authors' question, namely which of the contributing factors should be more prone to errors, is not likely to be resolved by the kind of data presented in their study. Obviously, a lower level of skill leads to a higher susceptibility for errors, but we have no evidence for a higher amount of genetic influence among the full AP possessors as opposed to the partial AP possessors. Additionally, it is important to note that the identification of neural correlates for AP is not equivalent with finding evidence for its innateness. One of the key findings of cognitive neuroscience concerns the neuroplasticity of the human brain, which is shaped by experience structurally as well as functionally. In the case of AP, we can use the electrophysiological as well as neuroimaging results to better understand the cognitive processes involved in the phenomenon. For many of the distinctive features in AP brains it is yet unclear, though, whether they are the cause for the development of AP or its consequence. 
While it is obvious that more research on behavioral as well as neural aspects of AP is needed to address the issue of AP development, the paper by Gruhn et al. clearly presents solid evidence for an influence of timbral manipulations on pitch labeling accuracy, as well as the impulse to further pursue potential influences on the development of this fascinating ability.

\section{ACKNOWLEDGMENTS}

This article was copyedited by Scott Bannister and layout edited by Diana Kayser.

\section{NOTES}

[1] Correspondence can be addressed to: Prof. Dr. Kathrin Bettina Schlemmer, Catholic University Eichstätt-Ingolstadt, Ostenstr. 26, D-85072 Eichstätt, kathrin.schlemmer@ku.de.

\section{REFERENCES}

Chin, C. (2003). The Development of Absolute Pitch: A Theory Concerning the Roles of Music Training at an Early Developmental Age and Individual Cognitive Style. Psychology of Music, 31(2), 155-171. https://doi.org/10.1177/0305735603031002292

Deutsch, D. (2013). Absolute pitch. In D. Deutsch (Ed.), The psychology of music (3rd ed.) (pp. 141-182). San Diego, CA, US: Elsevier Academic Press. https://doi.org/10.1016/B978-0-12-381460-9.00005-5

Elmer, S., Rogenmoser, L., Kühnis, J. \& Jäncke, L. (2015). Bridging the gap between perceptual and cognitive perspectives on absolute pitch. The Journal of Neuroscience, 35(1), 366-371. https://doi.org/10.1523/JNEUROSCI.3009-14.2015

Levitin, D. J. (1994). Absolute memory for musical pitch: Evidence from the production of learned melodies. Perception \& Psychophysics, 56(4), 414-423. https://doi.org/10.3758/BF03206733

Loui, P. (2016). Absolute pitch. In S. Hallam, I. Cross, \& M. Thaut (Eds.), The Oxford handbook of music psychology (2nd ed.) (pp. 81-94). New York, NY, US: Oxford University Press.

Marvin, E.W. \& Brinkman, A.R. (2000). The Effect of Key Color and Timbre on Absolute Pitch Recognition in Musical Contexts. Music Perception, 18(2), 111-137. https://doi.org/10.2307/40285905

Miyazaki, K. (1989). Absolute pitch identification: effects of timbre and pitch region. Music Perception, 7(1), 1-14. https://doi.org/10.2307/40285445

Miyazaki, K. (1990). The speed of musical pitch identification by absolute pitch possessors. Music Perception, 8, 177-188. https://doi.org/10.2307/40285495

Rakowski, A. \& Morawska-Büngeler, M. (1987). In search for the criteria of absolute pitch. Archives of Acoustics, 12(2), 75-87.

Rogenmoser, L., Elmer, S. \& Jäncke, L. (2015). Absolute pitch: Evidence for early cognitive facilitation during passive listening as revealed by reduced P3a amplitudes. Journal of Cognitive Neuroscience, 27(3), 623-637. https://doi.org/10.1162/jocn_a_00708

Schlemmer, K. B. (2009). Do absolute pitch possessors have a field independent cognitive style? In J. Louhivuori, T. Eerola, Suvi Saarikallio, T. Himberg \& P.-S. Eerola (Eds.), Proceedings of the 7th Triennial Conference of European Society for the Cognitive Sciences of Music (ESCOM 2009) Jyväskylä, Finland (pp. 480-486), http://urn.fi/URN:NBN:fi:jyu-2009411318. 
Schlemmer, K. B., Kulke, F., Kuchinke, L. \& van der Meer, E. (2005). Absolute pitch and pupillary response: Effects of timbre and key color. Psychophysiology, 42(2), 465-472. https://doi.org/10.1111/j.1469-8986.2005.00306.x

Schneider, P., \& Wengenroth, M. (2009). The neural basis of individual holistic and spectral sound perception. Contemporary Music Review, 28(3), 315 - 328. https://doi.org/10.1080/07494460903404402

Sergeant, D. C. (1969). Experimental investigation of absolute pitch. Journal of research in music education, 17(1), 135-143. https://doi.org/10.2307/3344200 